\title{
Analysis of surface EMG activation in hand percussion playing depending on the grasping type and the tempo
}

\author{
Hyun Ju Chong ${ }^{1, \dagger}$, Soo Ji Kim, ${ }^{2, t}$ Eun Kyoung Lee ${ }^{3}$, Ga Eul Yoo ${ }^{1, *}$ \\ 'Department of Music Therapy, Graduate School, Ewha Womans University, Seoul, Korea \\ 2Department of Music Therapy Education, Graduate School of Education, Ewha Womans University, Seoul, Korea \\ ${ }^{3}$ Venezia Orchestra, Seoul, Korea
}

\begin{abstract}
Although instrument playing-based training has been repeatedly reported to improve functional hand movements including grasping, the attempts to present quantitative information on physiological mechanism of grasping have been relatively insufficient to determine the type and the intensity of the exercises involved. This study aimed to examine the muscle activation during hand percussion playing depending on the grasping type and the playing tempo. A total of twelve healthy older adults with a mean age of $71.5 \mathrm{yr}$ participated in this study. Surface electrodes were placed on three grasping-related muscles: Flexor digitorum superficialis, extensor digitorum, and flexor pollicis brevis. Participants were instructed to play with the egg shaker, paddle drum mallet and clave involving different types of grasp at three different tempi (i.e., 80,100 , and $120 \mathrm{bpm}$ ) and sEMG data were collected during each playing. Significantly greater muscle activation was generated with the
\end{abstract}

small sphere type of egg shaker, compared to the handle type of paddle drum mallet and the small cylinder type of clave. Playing at faster tempo also elicited significantly greater muscle activation than at slower tempo. With regard to the rise time of muscle activation, while tempo significantly affected the rise time, the time to peak muscle activation did not significantly change depending on the grasping type. This study confirmed that grasping pattern and the tempo of movement significantly influence the muscular activation of grasping involved in instrument playing. Based on these results, clinical implication for instrument selection and structured instrument playing would be suggested.

Keywords: Electromyography, Grasping, Muscular contraction, Instrument playing

\section{INTRODUCTION}

A number of studies on age-related physical function has demonstrated that older adults show not only markedly declined capacity to generate force and maintain and control force production, but also increased force variability (Keogh et al., 2007; Ranganathan et al., 2001; Vaillancourt et al., 2003; Voelcker-Rehage and Alberts, 2005); and it is attributed to decreases in muscle mass and neural muscle activation (Sosnoff and Newell, 2006). Such weakened muscle strength is evident in the control of grasping forces. Deficits in the ability to accurately modulate grasping forces limit dexterous skills to manipulate target objects, which results in decline in motor performance related to daily living activities such as eating and dressing (Voelcker-Rehage and Alberts, 2005). Previous studies on the effects of practice-related strengthening training have demonstrated that these deficits in force production and control can be improved by repeated motor tasks involving of multitude of muscle groups (Keogh et al., 2007; Vaillancourt et al., 2003; Voelcker-Rehage and Alberts, 2005).

As the need for maintaining physical levels is increased in elderly population, it becomes significant for music therapists to make clinical decisions in designing applications meeting optimal physical functions for the population. As the field of music therapy expands based on the scientific evidences in regard to music and human behaviors, it becomes more important to understand the quantitative and qualitative relationships between therapeutic

\footnotetext{
${ }^{*}$ Corresponding author: Ga Eul Yoo

Department of Music Therapy, Graduate School, Ewha Womans University,

52 Ewhayeodae-gil, Seodaemun-gu, Seoul 03760, Korea

Tel: +82-2-3277-4232, Fax: +82-2-3277-6918, E-mail: bbird27@hotmail.com

${ }^{\dagger}$ Co-primary authors. Both authors contributed equally to this work.

Received: June 24, 2015 / Accepted: August 15, 2015
} 
goals and musical behaviors, which target the nonmusical goals. Instrument playing, as one of the standard music applications, primarily involves individuals' body movements and cognitive functioning. Because instrument playing can be effectively transferred to functional upper limb movements depending on the type, modification of the type and method of instrument playing has been tailored by professional music therapists to achieve specific goals. The effects of instrument playing-based training has been particularly evidenced in rehabilitative settings (Altenmüller et al., 2009; Chong et al., 2013; Han, 2011; Rojo et al., 2011; Schneider et al., 2007). Though, the focus has been placed primarily on functional changes after the intervention or training, rather than the systematic mechanism for the modulation of levels of stimulus including instrument type. In addition, the information that music therapists can deliver about instrument playing for improving physical functioning largely rely on visual observation of the playing patterns or on manipulating the weight of the instruments for developing muscle strength.

Although the effects of instrument playing-based training on grip force has also been evidenced (Schneider et al., 2007), there have been limited attempts to examine what contributes the outcome in terms of physiological mechanisms. Motor function with regard to grasping objects is affected by multiple factors. Multiple forearm and finger flexor and extensor muscles are involved in the grasping task, and the contribution of each muscle to the grasping force is affected by the required task-specific performance. Previous research findings have demonstrated that muscle activation involved in grasping differs by the mechanical properties of the objects grasped. Distinctive patterns of muscle activation were observed depending on the size, shape, length, and weight of objects (Brochier et al., 2004; Fujii et al., 2009; Fujisawa and Miura, 2010). These studies indicated that interaction between involved fingers and the area, as well as the pattern of contact with the surface of an object contribute to object-specific muscle activation, not just determined by a single force used to produce an expected motion or hand posture (Brochier et al., 2004). Furthermore, the posture of the upper limbs including the forearm (Mogk and Keir, 2003) and wrist (Li, 2002) while grasping objects determines the strength of the grasp.

Considering that instrument playing requires the ability to consistently grasp, hold, and lift different types of objects, which is enabled via stable force generation, analysis of the different patterns and amplitudes of muscular activation during instrument playing involving grasping is expected to present quantitative information for reference to aid in the selection of instruments and playing methods. In association with the final stage of motor command involved in motor generation, electromyography (EMG) data are indicative of muscular performance and forces (Duque et al., 1995; Hoozemans and van Dieën, 2005). Furthermore, it contributes to the determination of expected muscular movements and intensity of the exercises involved in a specific task. Therefore, this study aimed to examine the muscle activation while playing hand-percussion instruments depending on the type of the instrument, which requires different types of grasps and the tempo of playing answering following research questions:

1) Are there differences in forearm and finger sEMG activation depending on the grasping type and tempo?

2) Are there differences in the rise time of sEMG activation depending on the grasping type and tempo?

\section{MATERIALS AND METHODS}

\section{Participants}

Participants were recruited by advertising at local community centers for older adults. A total of twelve healthy older adults with a mean age of $71.5 \mathrm{yr}(\mathrm{SD}=7.0)$ were volunteered to participate in this study. Each participant was screened to ensure no history of neurological or psychiatric disorders. Participants were excluded if they had previous hand or finger injuries. All participants reported to have less than $5 \mathrm{yr}$ of music education or training. Ethical issues were reviewed and approved by Institutional Review Board of Ewha Womans University (IRB No. 62-9). An informed consent form was obtained from each participant prior to participation in this study. Demographic information is summarized in Table 1.

\section{Measurement instruments}

The EMG data for the forearm muscles were acquired via an eight-channel EMG system (Laxtha Inc., Korea). Three pairs of $\mathrm{Ag} / \mathrm{AgCL}$ surface electrodes (3M Inc., USA) were placed on three

Table 1. Characteristics of participants

\begin{tabular}{lc} 
& $\mathrm{n}=12$ \\
\hline Age $(\mathrm{M} \pm \mathrm{SD})$ & $71.5 \pm 7.0$ \\
Sex (Female/Male) & $12 / 0$ \\
Dominant Hand (Right/Left) & $12 / 0$ \\
$\mathrm{BM}^{\mathrm{a}}(\mathrm{M} \pm \mathrm{SD})$ & $25.5 \pm 2.9$ \\
Grip Power $^{b}(\mathrm{M} \pm \mathrm{SD})$ & $14.3 \pm 2.2$ \\
\hline
\end{tabular}

aBMl: Body Mass Index. The index was calculated by dividing bodyweight (kg) by height $(\mathrm{m})$ squared.

VValues for grip power $(\mathrm{kg})$ were obtained using Jamar Hydraulic Hand Dynamometer (5030J1, Preston). 
muscles of each participant's dominant hand: the flexor digitorum superficialis (FDS) as one of the main finger and wrist flexors; the extensor digitorum (ED), as one of the main finger and wrist extensors; and the flexor pollicis brevis (FPB), as the thumb abductor (Fig. 1). The ground electrode was attached to bony side of the back of the neck.

\section{Stimuli}

For the EMG measurement during instrument playing, three hand-percussion instruments, including the egg shaker, paddle drum mallet, and clave were selected, in that these are commonly used during playing-based intervention and involved a different type of grasp (Table 2). For the tempo condition, the tempi of 80 , 100 , and 120 beats per minute (bpm) were set and presented with the use of metronome.

\section{Procedures}

Experimental sessions were individually conducted in a quiet place in the community centers at which participants were recruited. Each participant was seated with her elbow flexed at approximately 90 degrees, forearm parallel to the ground, and wrist in a neutral position. Then the participant was instructed to grasp each

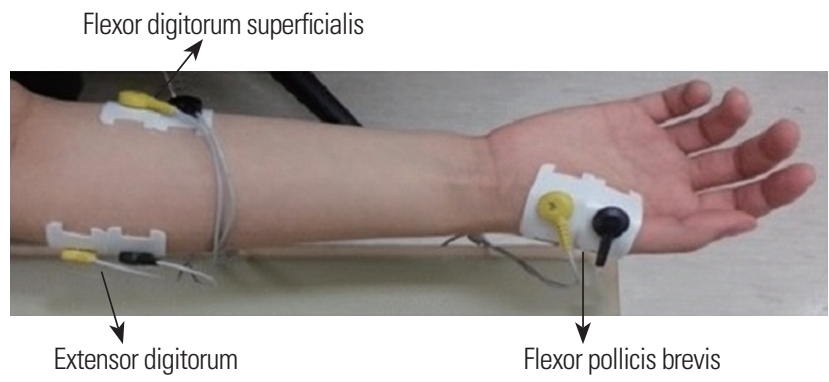

of three hand-percussion instruments with forearm in a pronated position and sound the instrument 10 times by lifting up and down (egg shaker), striking the paddle drum held by the investigator (paddle drum mallet), or striking the other pair held by the investigate (clave). Each participant was also asked to attempt to match the each of three tempi (i.e., 80, 100, $120 \mathrm{bpm}$ ) presented with the use of metronome. Each participant was entrained to the expected tempo while playing the instrument with the use of the metronome beat. Once the participant was confirmed to play the instrument at the presented tempo, the EMG activity was measured during playing each instrument without the use of the metronome beat. The order of task presentation (i. e., playing three instruments at three tempi) was randomly determined prior to the study. Between the trials, the participant was asked to pause for approximately 20 to $30 \mathrm{sec}$ to prevent forearm fatigue. After all of the playing tasks were administered, resting time was given until each participant was relaxed. Then the maximal voluntary contraction (MVC) for relevant muscles was obtained to normalize EMG data by asking each participant to produce maximum force against a stationary object grasped for five seconds.

\section{Data collection}

The EMG activity of the grasp-related muscles was collected during the percussive instrument playing tasks. Surface EMG signals were sampled at $1,024 \mathrm{~Hz}$. After the DC offset was removed, the signals were band-pass filtered at 20 to $450 \mathrm{~Hz}$. During each of 10 playing trials for each instrument, the root mean square (RMS) value of the signals was calculated and averaged. Then the percentage MVC value for each measured signal was analyzed to determine the differences among the instrument and tempo conditions.

Fig. 1. EMG electrode placement.

Table 2. Features of grasping each instrument

\begin{tabular}{|c|c|c|c|c|}
\hline Instrument & Diameter (cm) & Weight & Type of Grasp & Pattern of Finger Involvement \\
\hline Egg shaker & 4.2 & 29 & Small spherical & $\begin{array}{l}\text { High level of finger flexion and extension; the thumb's } \\
\text { opposition to other four fingers }\end{array}$ \\
\hline Paddle drum mallet & 0.9 & 58 & Hook & Less involvement of the thumb \\
\hline Clave & 2.54 & 45 & Small cylindrical & The thumb's opposition to other four fingers \\
\hline
\end{tabular}




\section{RESULTS}

\section{Muscular activation level}

The highest FDS activation data was observed during the egg shaker playing at $120 \mathrm{bpm}$, followed by when playing the same instrument at $100 \mathrm{bpm}$ and at $80 \mathrm{bpm}$. The least sEMG data was observed during the clave playing at $80 \mathrm{bpm}$. The sEMG data during egg shaker playing was found greater in all of the three tempo conditions than during the other two instruments playing. For all of three instruments, the highest sEMG activation was elicited at $120 \mathrm{bpm}$, followed by $100 \mathrm{bpm}$. The least muscle activation was observed at $80 \mathrm{bpm}$. The EMG data of each instrument at each tempo were summarized in Table 3.

A two-way repeated measures ANOVA was conducted to examine the differences in the sEMG data depending on the grasping type and the tempo. There were significant differences in the mean amplitudes (\%MVC) in FDS depending on the grasping type, $F(2$, $22)=9.612, P=0.001$, and the tempo, $F(2,22)=5.706, P=0.026$. There was no significant interaction between the grasping type and the tempo factors, $F(4,44)=1.200, P=0.317$ (Fig. 2). Post hoc paired comparisons with the Bonferroni correction showed that egg shaker grasping elicited a significantly greater level of muscle activation than both the paddle drum mallet $(P=0.033)$ and the clave grasping $(P=0.033)$. The differences in the mean EMG amplitude between the paddle drum mallet and the clave conditions did not reach statistical significance $(P=0.487)$. In terms of the tempo, the mean EMG amplitude during playing at $120 \mathrm{bpm}$ was significantly higher than during playing at $80 \mathrm{bpm}$ $(P=0.038)$ and during playing at $100 \mathrm{bpm}(P=0.001)$. There was no significant difference in the muscle activation between playing

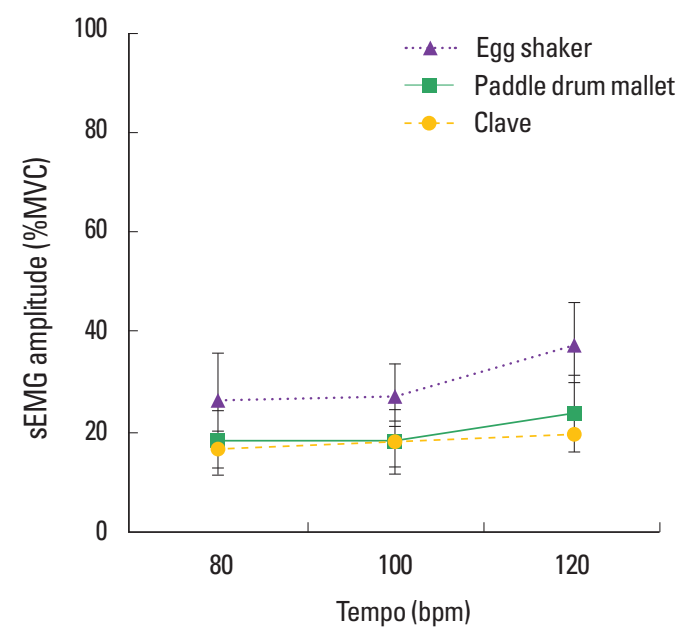

Fig. 2. FDS activation depending on the grasping type and tempo conditions. at $80 \mathrm{bpm}$ and at $100 \mathrm{bpm}(P=0.749)$.

For ED, the highest muscle activation was elicited during the egg shaker playing at $120 \mathrm{bpm}$. The sEMG data during egg shaker playing at $100 \mathrm{bpm}$ and $80 \mathrm{bpm}$ were also greater than during the other two instruments playing at all levels of playing tempo. The least EMG data was observed during the clave playing at $80 \mathrm{bpm}$. All of three instruments showed the highest EMG activation at $120 \mathrm{bpm}$, then followed by $100 \mathrm{bpm}$ and $80 \mathrm{bpm}$. The descriptive information of muscle activation in each condition (grasping type and tempo) was summarized in Table 4.

A two-way repeated measures ANOVA results showed that there were significant differences in the ED activation value (\%MVC) depending on the grasping type, $F(2,22)=4.205, P=0.028$, and the tempo, $F(2,22)=6.875, P=0.013$. There was no significant interaction between the grasping type and the tempo factors, $F(4$, 44 ) $=0.537, P=0.592$ (Fig. 3). Post hoc paired comparisons demonstrated that a significantly higher level of muscle activation was elicited during egg shaker grasping than during the paddle drum mallet $(P=0.040)$ and the clave grasping $(P=0.048)$. There was no significant difference between the paddle drum mallet and the clave conditions $(P=0.929)$. With regard to the tempo, playing

Table 3. sEMG value (\%MVC) depending on the grasping type in flexor digitorum superficialis (FDS)

\begin{tabular}{lrrrrrrrrr}
\hline \multirow{2}{*}{ Grasping type } & \multicolumn{2}{c}{ Tempo 80} & & \multicolumn{2}{c}{ Tempo 100} & & \multicolumn{2}{c}{ Tempo 120 } \\
\cline { 2 - 3 } \cline { 8 - 9 } & M & SD & & M & SD & & M & SD \\
\hline Egg shaker & 27.86 & 21.14 & & 27.99 & 12.56 & & 38.56 & 15.70 \\
Paddle drum mallet & 18.25 & 13.51 & & 18.61 & 12.42 & & 24.31 & 15.14 \\
Clave & 16.45 & 7.13 & & 18.01 & 8.53 & & 19.74 & 6.65 \\
\hline
\end{tabular}

Table 4. sEMG value (\%MVC) depending on the grasping type in extensor digitorum (ED)

\begin{tabular}{lcccccccc}
\hline \multirow{2}{*}{ Grasping type } & \multicolumn{2}{c}{ Tempo 80} & & \multicolumn{2}{c}{ Tempo 100} & & \multicolumn{2}{c}{ Tempo 120 } \\
\cline { 2 - 3 } & M & SD & & M & SD & & M & SD \\
\hline Egg shaker & 28.77 & 27.37 & & 33.43 & 25.83 & & 34.76 & 19.55 \\
Paddle drum mallet & 20.93 & 12.06 & & 22.18 & 14.08 & & 24.56 & 13.05 \\
Clave & 19.43 & 10.99 & & 22.18 & 14.42 & & 26.85 & 17.19 \\
\hline
\end{tabular}

Table 5. sEMG value (\%MVC) depending on the grasping type in flexor pollicis brevis (FPB)

\begin{tabular}{|c|c|c|c|c|c|c|}
\hline \multirow{2}{*}{ Grasping type } & \multicolumn{2}{|c|}{ Tempo 80} & \multicolumn{2}{|c|}{ Tempo 100} & \multicolumn{2}{|c|}{ Tempo 120} \\
\hline & M & SD & $M$ & SD & $M$ & SD \\
\hline Egg shaker & 25.04 & 26.47 & 23.92 & 19.28 & 39.34 & 36.74 \\
\hline Paddle drum mallet & 16.17 & 21.08 & 16.31 & 18.90 & 21.29 & 27.73 \\
\hline Clave & 13.07 & 12.15 & 14.11 & 14.23 & 16.77 & 13.49 \\
\hline
\end{tabular}




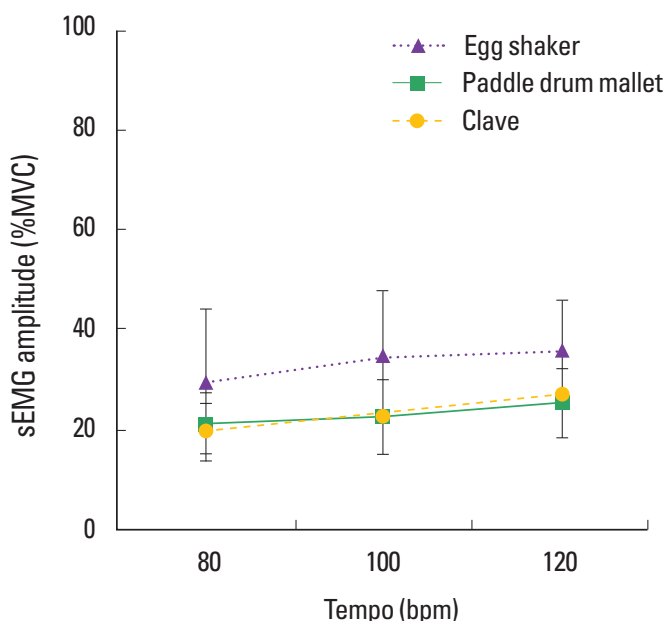

Fig. 3. ED activation depending on the grasping type and tempo conditions.

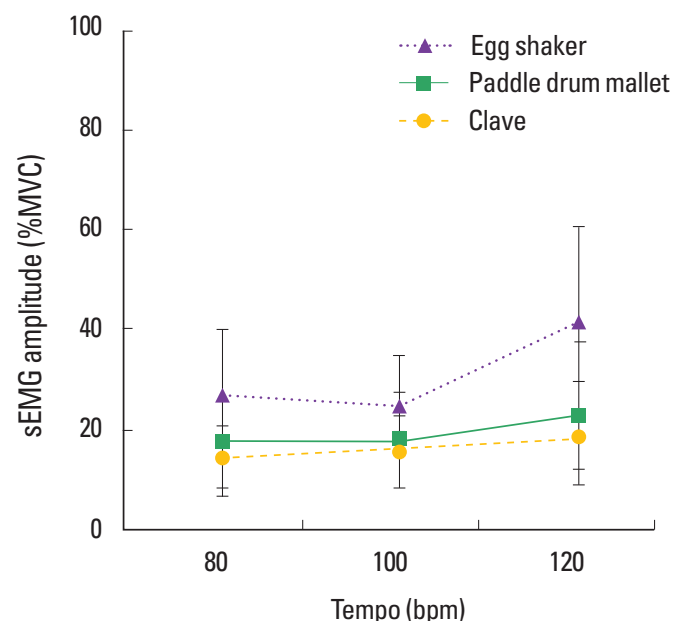

Fig. 4. FPB activation depending on the grasping type and tempo conditions.

Table 6. Rise time (ms) in muscle activation depending on the grasping type

\begin{tabular}{|c|c|c|c|c|c|c|c|}
\hline \multirow{2}{*}{ Muscle } & \multirow{2}{*}{ Instrument to be grasped } & \multicolumn{2}{|c|}{ Tempo 80} & \multicolumn{2}{|c|}{ Tempo 100} & \multicolumn{2}{|c|}{ Tempo 120} \\
\hline & & M & SD & M & SD & M & SD \\
\hline \multirow[t]{3}{*}{ FDS } & Egg shaker & 152 & 64 & 143 & 41 & 131 & 51 \\
\hline & Paddle drum mallet & 178 & 72 & 159 & 76 & 135 & 14 \\
\hline & Clave & 198 & 71 & 152 & 45 & 143 & 42 \\
\hline \multirow[t]{3}{*}{ ED } & Egg shaker & 205 & 116 & 197 & 49 & 193 & 54 \\
\hline & Paddle drum mallet & 228 & 118 & 209 & 115 & 163 & 53 \\
\hline & Clave & 236 & 108 & 194 & 86 & 174 & 73 \\
\hline \multirow[t]{3}{*}{ FPB } & Egg shaker & 190 & 49 & 185 & 87 & 164 & 68 \\
\hline & Paddle drum mallet & 243 & 112 & 213 & 82 & 160 & 72 \\
\hline & Clave & 237 & 81 & 220 & 97 & 178 & 80 \\
\hline
\end{tabular}

at $80 \mathrm{bpm}$ elicited significantly lower mean EMG amplitudes than at $100 \mathrm{bpm}(P=0.006)$ and during playing at $120 \mathrm{bpm}(P=0.007)$. There was no significant difference in the muscle activation between playing at $100 \mathrm{bpm}$ and at $120 \mathrm{bpm}(P=0.153)$.

With regard to the primary thumb flexor, FPB, the sEMG data during the egg shaker playing at $120 \mathrm{bpm}$ was the highest, followed by playing at $100 \mathrm{bpm}$ and at $80 \mathrm{bpm}$. As in other muscles, the least EMG data was observed during the clave playing at $80 \mathrm{bpm}$. In all of three grasping type conditions, while the fastest playing tempo (i.e., $120 \mathrm{bpm}$ ) led to the highest EMG activation, the slowest playing tempo (i.e., $80 \mathrm{bpm}$ ) elicited the least muscle activation. The information on the EMG data collected was displayed in Table 5.

The results of a two-way repeated measures ANOVA showed significant differences were found in level of the FPB activation (\%MVC) depending on the grasping type, $F(2,22)=6.851$, $P=0.005$, and the tempo, $F(2,22)=9.266, P=0.006$. There was no significant interaction between the instrument and the tempo factors, $F(4,44)=1.948, P=0.161$ (Fig. 4). Post hoc paired comparison results showed that during egg shaker playing, significantly greater muscle activation was elicited than during the paddle drum mallet $(P=0.005)$ and the clave playing $(P=0.016)$. No significant difference in the sEMG value was found between the paddle drum mallet and the clave conditions $(P=0.419)$. In terms of the tempo, playing at $120 \mathrm{bpm}$ was found with a significantly higher sEMG level than playing at $80 \mathrm{bpm}(P=0.009)$ and playing at $100 \mathrm{bpm}(P=0.009)$. The difference in the muscle activation between playing at $80 \mathrm{bpm}$ and at $100 \mathrm{bpm}$ was not statistically significant $(P=0.984)$.

\section{Rise time in muscle activation}

In terms of rise time in three muscles, the time to the peak muscle activation was the shortest during playing egg shaker at each tempo. The longest time to the peak was observed during clave 
playing at each of three tempi. For all three instruments, faster tempo condition reached to the peak activation with less time (Table 6).

Further analyses demonstrated that the identical patterns were observed across grasping type conditions. For FDS, a two-way repeated measured ANOVA showed that while there was no significant effect of the grasping type, $F(2,22)=1.135, P=0.339$, the factor of the tempo significantly affected the rise time in muscle activation during playing each of three instruments, $F(2,22)$ $=3.992, P=0.033$. Interaction between the grasping type and tempo factors was not statistically significant $F(4,44)=0.636$, $P=0.640$. In terms of the effect of the playing tempo, post hoc paired comparisons showed that a significant difference was found between playing at $120 \mathrm{bpm}$ and at $80 \mathrm{bpm}(P=0.007)$. The other paired comparisons with regard to the tempo factor did not reach statistical significance. With regard to $\mathrm{ED}$, the results of a two-way repeated measured ANOVA showed that the effects of both the grasping type and the playing tempo were not statistically significant. A two-way repeated measure ANOVA results in FPB were similar to those in FDS. While there is no significant effect of the grasping type, $F(2,22)=1.167, P=0.330$, the effect of the playing time was significant, $F(2,22)=4.270, P=0.027$. Interaction between the grasping type and the playing tempo factors did not reach statistical significance, $F(4,44)=0.366, P=0.831$, indicating that the changes in FPB activation depending on the playing time were consistent at each tempo.

\section{DISCUSSION}

First, this study showed that muscular activation significantly differed in relation to the different grasps required to play hand-percussion instruments. Significantly greater muscle activation was generated with the small sphere-type egg shaker, compared to the handle-type paddle drum mallet and the small cylinder-type clave in all of three muscles: FDS, ED, and FPB (Table 7). This is consistent with previous research findings, which demonstrated distinctive object-specific muscle activation in relation to the shape of the hands and fingers needed to appropriately adapt to the physical features of objects (Brochier et al., 2004). It is also related to the research findings that the degree of synergic and counteractive interaction across fingers, which is a primary contributing factor to force exertion, differs depending on the type of object grasped (Kong and Lowe, 2005). In addition, this study results were explained with highest EMG data in the spherical grasp (i.e., egg shaker), where the highest degree of thumb flexion and extension are involved and, accordingly, lead to highest grasping forces (Lin et al., 2011).
Table 7. sEMG patterns depending on the instrument to be grasped

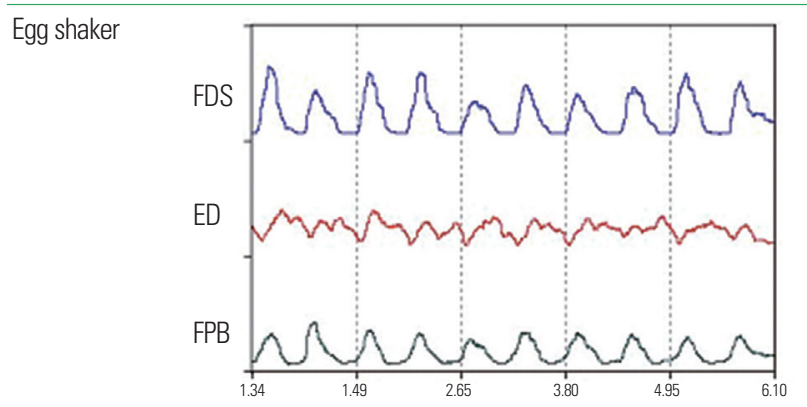

Paddle drum

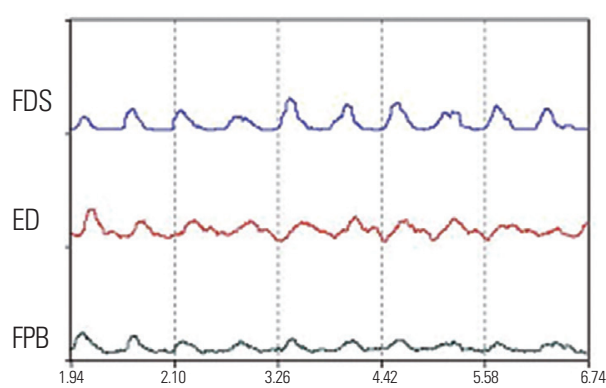

Clave

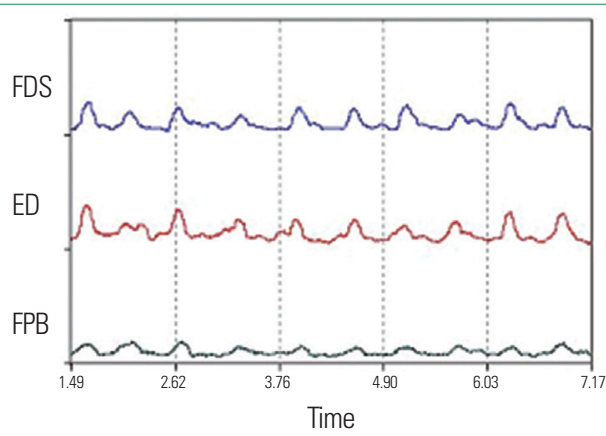

It might be attributed to the fact that the thumb plays a critical role in exerting end-point forces in grasping and maintaining constant grasping by opposing other fingers.

Furthermore, playing at a faster tempo elicited greater muscle activation than at a slower tempo. Previous research demonstrated that increased tempo led to significantly greater muscular activity, in an attempt to ensure the precise motor performance despite increasing demand on the spatial accuracy of the planned motion (Furuya et al., 2012). Grasping force is also increased at a greater speed to maintain safety and stability of the objects held (Kinoshita et al., 1996).

In terms of rise time of muscle activation, no significant effects were found depending on the grasping type in all of three muscles. Meanwhile, the rise time significantly decreased with faster tempo. It indicates that the time to peak muscle contraction may 
be maintained constantly across the instruments to be grasped. It can be also explained that the grasping type, which involve muscle groups in different ways, would lead to the difference in the level of muscle activation, but would not affect time-dependent features of muscle activation.

As indicated by previous studies, the EMG data of the engaged muscles are predictive of grasping forces (Hoozemans and van Dieën, 2005). Based on these results, clinical implications for grasp-related instrument playing could be suggested. Considering that deficits in the ability to accurately modulate grasping forces are attributed to decreases in muscle mass and neural muscle activation (Ranganathan et al., 2001; Sosnoff and Newell, 2006; Vaillancourt et al., 2003), increasing muscle firing rate, despite the decline in neural drive (i.e., functioning motor units and motor unit firing rate) of motor neuron observed with aging (Sosnoff and Newell, 2006) could be a key factor. The literature has indicated that deficits in force production and control can be improved by performing repeated motor tasks involving a multitude of muscle groups (Keogh et al., 2007; Vaillancourt et al., 2003; Voelcker-Rehage and Alberts, 2005). Accordingly, the results of this study indicate that the grasping pattern and position of the arm, hand, and finger muscles significantly influenced the force and efficiency of grasping in this population. Selection of instrument along with proper playing methods is critical in music therapy for improving upper limb movement. By identifying the level of muscle activation induced by three simple hand percussion instruments, the findings of this study provide practical information to assist in the clinical decision-making of music therapists. Future studies should be continued to provide more systematic information in regard with musical behaviors.

\section{CONFLICT OF INTEREST}

No potential conflict of interest relevant to this article was reported.

\section{ACKNOWLEDGEMENTS}

This work was supported by the National Research Foundation of Korea Grant funded by the Korean Government (NRF-20121788-001-2).

\section{REFERENCES}

Altenmüller E, Marco-Pallares J, Münte T, Schneider S. Neural reorganization underlies improvement in stroke-induced motor dysfunction by music-supported therapy. Annals N Y Acad Sci 2009;1169:395-405.

Brochier T, Spinks RL, Umilta MA, Lemon RN. Patterns of muscle activity underlying object-specific grasp by the macaque monkey. J Neurophysiol 2004;92:1770-1782.

Chong HJ, Cho SR, Jeong EJ, Kim SJ. Finger exercise with keyboard playing in adults with cerebral palsy: A preliminary study. J Exer Rehabil 2013;9:420-425.

Duque J, Masset D, Malchaire J. Evaluation of handgrip force from EMG measurements. Appl Ergon 1995;26:61-66.

Fujii S, Kudo K, Shinya M, Ohtsuki T, Oda S. Wrist muscle activity during rapid unimanual tapping with a drumstick in drummers and nondrummers. Motor Control 2009;13:237-250.

Fujisawa T, Miura M. Investigating a playing strategy for drumming using surface electromyograms. Acoust Sci Technol 2010;31:300-303.

Furuya S, Aoki T, Nkahara H, Kinoshita H. Individual differences in the biomechanical effect of loudness and tempo on upper-limb movements during repetitive piano keystrokes. Hum Mov Sci 2012;31:26-39.

Han S. The effect of using therapeutic instrumental music performance (TIMP) in uninvolved hand function for children with spastic cerebral palsy. J Music Hum Behav 2011;8:1-16.

Hoozemans MJ, van Dieën JH. Prediction of handgrip forces using surface EMG of forearm muscles. J Electromyogr Kinesiol 2005;15:358-366.

Kinoshita H, Kawai S, Ikuta K, Teraoka T. Individual finger forces acting on a grasped object during shaking actions. Ergonmics 1996;39: 243256.

Keogh JW, Morrison M, Barrett R. Strength training improves the tri-digit finger-pinch force control of older adults. Arch Phys Med Rehabil 2007; 88:1055-1063.

Kong Y, Lowe BD. Evaluation of handle diameters and orientations in a maximum torque task. Int J Ind Ergon 2005;35:1073-1084.

Li Z. The influence of wrist position on individual finger forces during forceful grip. J Hand Surg 2002;27:866-896.

Lin H, Kuo L, Liu H, Wu W, Su F. The three-dimensional analysis of three thumb joints coordination in activities of daily living. Clin Biomech 2011;26:371-376.

Mogk J, Keir P. The effects of posture on forearm muscle loading during gripping. Ergonomics 2003;46:956-975.

Ranganathan VK, Siemionow V, Sahgal V, Yue GH. Effects of aging on hand function. J Am Geriatr Soc 2001;49:1478-1484.

Rojo N, Amengual J, Juncadella M, Rubio F, Camara E, Marco-Pallares J, Schneider S, Veciana M, Montero J, Mohammadi B, Altenmüller E, Grau C, Münte TF, Rodriguez-Fornells A. Music-supported therapy induces plasticity in the sensorimotor cortex in chronic stroke: a single-case study using multimodal imaging (fMRI- TMS). Brain Inj 2011;25:787-793. 
Schneider S, Schönle PW, Altenmüller E, Münte TF. Using musical instruments to improve motor skill recovery following a stroke. J Neurol 2007;254:1339-1346.

Sosnoff JJ, Newell KM. Are age-related increases in force variability due to decrements in strength? Exp Brain Res 2006;174:86-94.
Vaillancourt DE, Larsson L, Newell KM. Effects of aging on force variability, single motor unit discharge patterns, and the structure of 10,20 , and $40 \mathrm{~Hz}$ EMG activity. Neurobiol Aging 2003;24:25-35.

Voelcker-Rehage C, Alberts JL. Age-related changes in grasping force modulation. Exp Brain Res 2005;166:61-70. 